\title{
Large Parapharyngeal Mass: A Challenging Differential Diagnosis
}

\author{
Massimo Ralli ${ }^{1}$, Marco de Vincentiis ${ }^{2}$, Antonio Greco ${ }^{2}$
}

1. Department of Oral and Maxillofacial Sciences, Sapienza University of Rome, Italy.

2. Department of Sense Organs, Sapienza University of Rome, Italy.

Corresponding Author: Massimo Ralli, MD, $\mathrm{PhD}$

Address: Department of Oral and Maxillofacial Sciences, Sapienza University of Rome. Viale del Policlinico 155, 00186, Rome Italy.

Email: massimo.ralli@uniroma1.it - Telephone: +390649976808

\section{Case Description}

A 35-year-old man presented at the Otolaryngology Department of the Sapienza University or Rome in February 2017 with a 14-month history of breathing difficulties and dysphagia. Endoscopic examination revealed a bulking in the right parapharyngeal space, extending into the oropharynx, dislocating the pharyngeal wall and causing a noticeable obstruction of respiratory tract; no externally visible cervical mass was present. Laboratory investigations and radiography of the chest were normal.

Contrast-enhanced Computed Tomography (CT) scan revealed a large mass centered in the right parapharyngeal space causing medialization of the pharyngeal wall. A contrast-enhanced Magnetic Resonance Imaging (MRI) scan demonstrated a T2-hyperintense and enhancing lesion measuring $74 \times 52 \times 36 \mathrm{~mm}$ centered in the right parapharyngeal space with relatively well-defined, smooth margins and some peripheral calcifications (Figures A and B). No lymph node involvement was detected by CT and MRI. The preoperative differential diagnosis centered on pleomorphic 
adenoma, vagal nerve schwannoma, paraganglioma, carcinoma ex pleomorphic adenoma, or lymphoma.

The mass was surgically removed through a transcervical dissection approach with mandibulotomy for better exposure of the neoplasm; careful sharp and blunt dissection allowed complete excision of the mass. No reactive adenopathies were found during surgery. No intraoperative complications occurred. Histological examination showed scattered foci of malignant transformation characterized by nuclear pleomorphism, frequent atypical mitotic figures, hemorrhage, and necrosis consistent with adenocarcinoma ex pleomorphic adenoma, an uncommon entity with significant clinical and pathological relevance. Follow-up endoscopy performed 3 months after surgery showed no persistence of the disease; patient reported no residual symptoms and no post-surgery complications.

Tumors of the parapharyngeal space are rare entities that represent less than $1 \%$ of all head and neck neoplasms. Parapharingeal masses may represent a relevant differential diagnostic challenge for clinicians. The common benign character of these conditions does not exclude malignancy, such as in this case in which malignant transformation was observed.

\section{Figure Legend}

Figure: Magnetic Resonance Imaging (MRI) showing a large parapharyngeal mass. (A) coronal section of contrast-enhanced MRI showing a T2-hyperintense and enhancing lesion in the right parapharyngeal space with relatively well-defined, smooth margins and some peripheral calcifications (white arrow). This image shows an evident dislocation of the pharyngeal wall that causes a noticeable obstruction of respiratory tract confirming the breathing difficulties and dysphagia symptoms reported by the patient. (B) axial section of MRI shows extension of the mass to the oropharynx (white arrows). 


\section{Clinical Question}

Patient with a large MRI contrast-enhancing mass with well-defined margins and peripheral calcifications centered in the right parapharyngeal space causing breathing difficulties and dysphagia. What is the diagnosis?

1. pleomorphic adenoma

2. vagal nerve schwannoma

3. carcinoma ex pleomorphic adenoma *

4. lymphoma

\section{Contributors section}

MR wrote the manuscript draft and performed literature search. MdV contributed to data interpretation and manuscript correction. AG contributed to manuscript correction and figure editing. All authors were equally involved in clinical diagnosis and surgical management of the patient. All authors have approved the final version of the manuscript. Written informed consent to publication was obtained.

\section{Conflict of interest statement}

The authors declared no conflicts of interest. 